\title{
Observations of a bright plume in solar granulation
}

\author{
R. I. Kostik ${ }^{1}$ and E. V. Khomenko ${ }^{2,1}$ \\ 1 Main Astronomical Observatory, NAS, 03680 Kyiv, Zabolotnogo str. 27, Ukraine \\ 2 Instituto de Astrofísica de Canarias, 38205, C/ Vía Láctea, s/n, Tenerife, Spain \\ e-mail: khomenko@iac.es
}

Received 24 January 2007 / Accepted 23 July 2007

\section{ABSTRACT}

\begin{abstract}
Aims. The aim of this paper is to analyze the thermal properties, oscillatory, and flow motions of a bright, long-lasting feature observed in solar granulation, which we call the plume.

Methods. We used the spectral observations of quiet granulation at solar disc center, including the two Fe II 5234 and Fe I $6393 \AA$ lines recorded simultaneously at the German Vacuum Tower telescope in Tenerife. The recorded data revealed a stable, bright structure of 3-4 arcsec size present during the whole $2.5 \mathrm{~h}$ of observations. We compare the velocity fields extracted by means of a $\lambda$-meter method and temperature and pressure stratification obtained after inversion of the profiles related to granules, intergranular lanes, and the plume.

Results. The following results were obtained: (i) the correlation between variations in convective velocity and intensity in the plume is close to zero at all observed heights; (ii) the velocity flow in the plume changes from a downflow in the deep layers to an upflow in the upper layers; (iii) the brightness of the plume increases with height; (iv) the amplitudes of the five-minute oscillations of intensity and velocity are twice lower in the plume than outside, and vertically propagating waves are observed; (v) the plume is hotter and denser than the quiet Sun in the upper photosphere.

Conclusions. We conclude that the observed phenomenon has a non-convective origin. The decrease in the amplitudes of oscillations in the plume cannot be attributed to the higher density in comparison to the surrounding atmosphere. Along with other findings, this indicates the possible presence of magnetic field.
\end{abstract}

Key words. Sun: photosphere - Sun: granulation - Sun: oscillations - Sun: magnetic fields

\section{Introduction}

The spectra of solar granulation comprise many scales and lifetimes starting from supergranular cells occupying $20 \mathrm{Mm}$ and remaining virtually stable for days (Leighton et al. 1962), down to mesogranulation (about $5 \mathrm{Mm}$ size and a lifetime of hours, see November et al. 1981; Deubner 1989), and granulation (with a typical size of about 1 arcsec and lifetime of minutes, see Title et al. 1989). The magnetic field plays an important role in the process of solar convection and in the appearance of granulation. Granules in active regions or close to them show different statistical properties from those in the quiet regions (see e.g., Sobotka et al. 1994). In the regions of enhanced magnetic flux, such as the borders of supergranules, bright points are observed in the centers of intergranular lanes (seen with paricularly high contrast in G-band, e.g., Shelyag et al. 2004; Stein et al. 2004). Sometimes a small pore is formed inside a downflowing region (see an example in Berger \& Title 2001) that can grow into a sunspot or disappear after a short time.

Magnetic features observed in the solar granulation have different contrasts depending on their size and the value of the magnetic field, as well as on the spectral range and height in the atmosphere (Spruit \& Zwaan 1981). Small-scale magnetic flux tubes manifest themselves as bright points of sub-arcsec size in G-band filtergrams (related to lower photospheric layers) and also appear bright in, e.g., Ca II H filtergrams (Berger et al. 2004) or in the photospheric line core images (both related to higher photospheric layers) due to the line gap effect (e.g., Beckers \& Schröter 1968; Stangl \& Hirzberger 2005). Features with larger sizes of 3-4 arcsec, such as pores, appear dark in the photospheric observations. However, under insufficient resolution one also observes large bright features, such as conglomerates of flux tubes, apparently seen as a single bright structure (Soltau 1997). The lifetime of all these features depends on their size and magnetic flux and changes from minutes to hours and days.

The present paper is dedicated to a long-lasting bright feature observed in the quiet solar granulation. During spectral observations of a quiet area near the disc center, performed in 2001 at the German VTT at the Observatorio del Teide (Tenerife), a suspicious feature was detected that persisted during the whole time series of almost $3 \mathrm{~h}$ duration. The feature was brighter than the local granulation at heights of $200-500 \mathrm{~km}$, but was not distinguishable at the continuum level. Its horizontal size was about 3 arcsec, thus exceeding the typical sizes of a granule, being closer to the size of a small pore. The flow inside the feature was directed downward in the deep layers. The purpose of our study is to investigate the velocity and intensity fields observed in this feature at different photospheric heights and to recover its thermal conditions in order to clarify its physical origin. In the rest of the paper, for convenience, we call this feature "the plume".

\section{Observations}

The data were taken in 2001 at the German Vacuum Tower Telescope at the Observatorio del Teide (Tenerife). The spectra of the Fe I 6393.6 and Fe II $5324 \AA$ lines were recorded simultaneously in a quiet area close to the solar disc center with a 

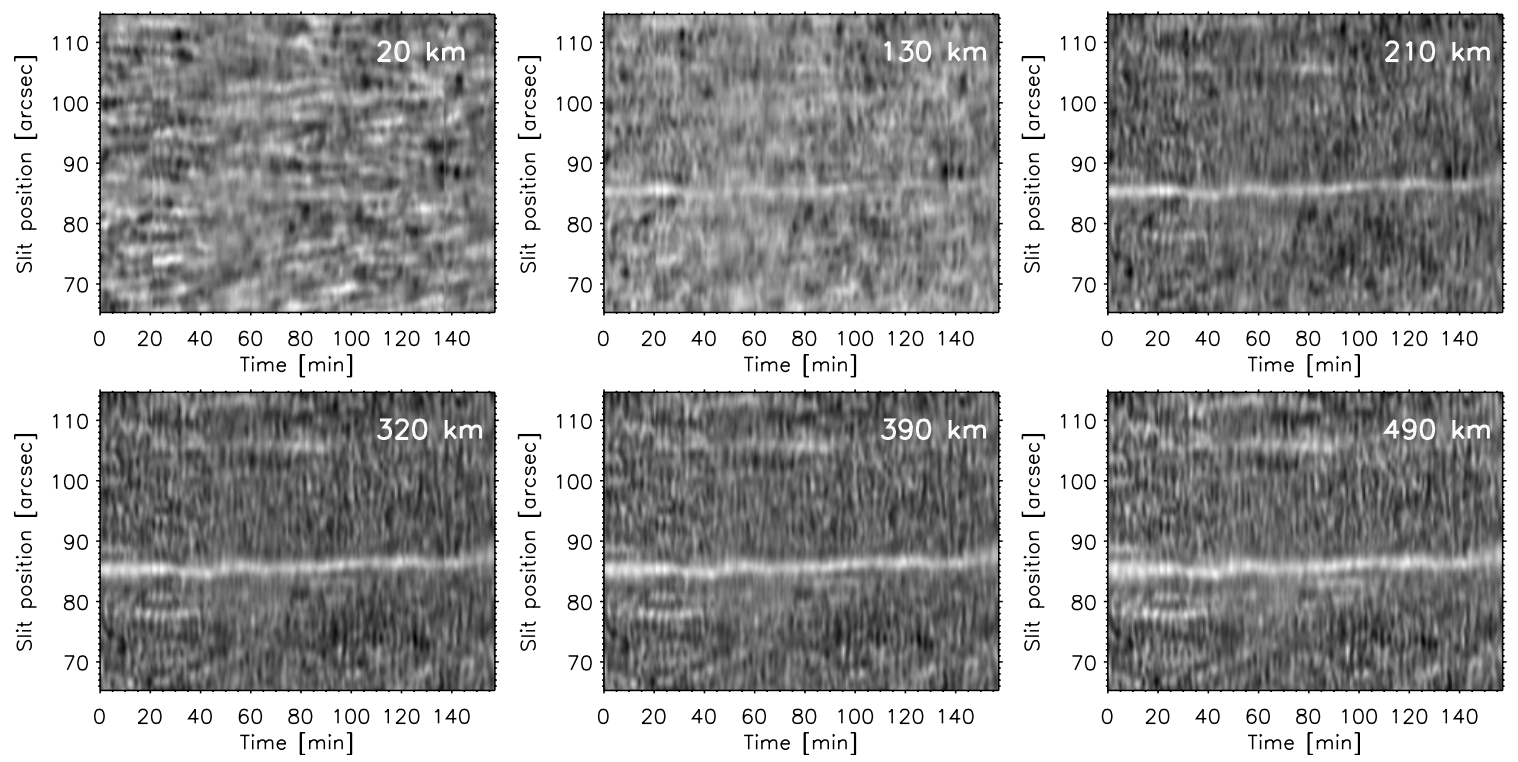

Fig. 1. Time-slice diagrams of the intensity variations at several heights measured from the Fe I $6393 \AA$ Aine profiles. The plume appears as a bright feature at the slit position $\approx 85$ arcsec. Note the absence of any particular details at the position of the plume at the bottom layers $(20 \mathrm{~km})$.

Table 1. Parameters of the observed lines.

\begin{tabular}{ccccccc}
\hline \hline $\begin{array}{c}\lambda \\
{[\AA]}\end{array}$ & Elem. & $\begin{array}{c}\text { EPL } \\
{[\mathrm{eV}]}\end{array}$ & $\log g f$ & $D$ & $\begin{array}{c}W \\
{[\mathrm{pm}]}\end{array}$ & $\begin{array}{c}H_{D} \\
{[\mathrm{~km}]}\end{array}$ \\
\hline 6393.61 & Fe I & 2.42 & -1.55 & 0.745 & 12.8 & 570 \\
5234.62 & Fe II & 3.22 & -2.45 & 0.722 & 8.8 & 370 \\
\hline
\end{tabular}

fixed slit position. The parameters of the observed lines are given in Table 1. The values of the excitation potential of the lower level EPL, oscillator strength $\log g f$, line depth $D$, equivalent width $W$, and height of formation of the line core $H_{D}$ were taken from Gurtovenko \& Kostik (1989). Both these lines are rather strong, unblended and they form at different depths in the solar atmosphere and have different temperature sensitivities. Thus, their simultaneous registration allows for more reliable information about the height dependence of granular and oscillatory motions.

The duration of the time series was 158 min with a $10 \mathrm{~s}$ interval between the successive exposures (943 images in total). The size of the spectrograph slit corresponded to 140 arcsec. As detectors we used two identical CCD cameras with $2048 \times 2048$ pixels in a two-by-two binning mode. The binned pixel size was $0 . ' 136$ and the slit width was $80 \mu \mathrm{m}$ (equivalent to 0.4$)$.

The image of the observed area was kept on the slit using a correlation tracker guiding on a small nearby pore. This has allowed the image motion on the spectrograph slit to be reduced considerably. During the observation period it did not exceed 0.'4. The seeing conditions were rather stable during the duration of the observations. The contrast of granulation at the continuum wavelength (after reduction of the images) was 4-5\% during the first hour of observations and a bit weaker during the rest of the time. In order to check the presence of magnetic network patches or other manifestations of magnetic activity, Ca II K slit-jaw images were taken simultaneously to the observations. The quality of the recorded slit-jaw images unfortunately did not allow us to confirm or discard this possibility. The spatial resolution of the observations estimated from the power spectra of the spatial variation at the continuum wavelength was not better than 1 arcsec.

Following a standard procedure, all 943 spectra of both lines were corrected for the dark current, flat field, and variations in the transparency of the earth atmosphere during the time of the observations. Using a $\lambda$-meter technique (for a detailed description of the method see Stebbins \& Goode 1987), we calculated the variations in line intensity and velocity at 11 levels of the line profiles, which correspond to 11 heights in the solar atmosphere. In order to ascribe an approximate formation height to each intensity level, radiative transfer calculations were performed for the Fe I 6393 and Fe II $5234 \AA$ lines in a standard solar model atmosphere MACKKL (Maltby et al. 1986) with the help of the NATAJA code (Shchukina \& Trujillo Bueno 2001; Shchukina 2004, private communication). It should be noted, however, that the formation heights can vary widely depending on the temperature structure at the observed point (e.g., in granules and intergranular lanes); thus, this information is used for illustration purposes. In general it is correct to assume that when approaching the line core, the photons come from successively higher layers of the solar atmosphere.

The following parameters are defined for each time moment $i$ and slit position $j$ : variations in the intensity with respect to the mean value at the corresponding level $l$ in units of the mean continuum intensity $I_{\mathrm{c}}, \delta I_{l}(i, j)$ and the line-of-sight (LOS) velocity variations at each level $l$ with respect to the zero velocity position $\delta V_{l}(i, j)$. An absolute wavelength calibration was not possible due to the absence of telluric spectral lines in the observed spectral window. An approximate calibration was achieved by considering the zero velocity position as the one of the average profile over the whole space and time. This position may contain a residual blue shift.

While inspecting the time-slice diagrams of the intensity field at heights $130-490 \mathrm{~km}$, we found a bright feature that existed during the whole period of observations. An example of such diagrams at several heights in the photosphere extracted from the Fe I $6393 \AA$ line is shown in Fig. 1. The plume appears as a bright feature of 3-4 arcsec size at the slit position of 85 arcsec already at a height of $130 \mathrm{~km}$. Its position is stable with no significant horizontal motions of the feature. The intensity 

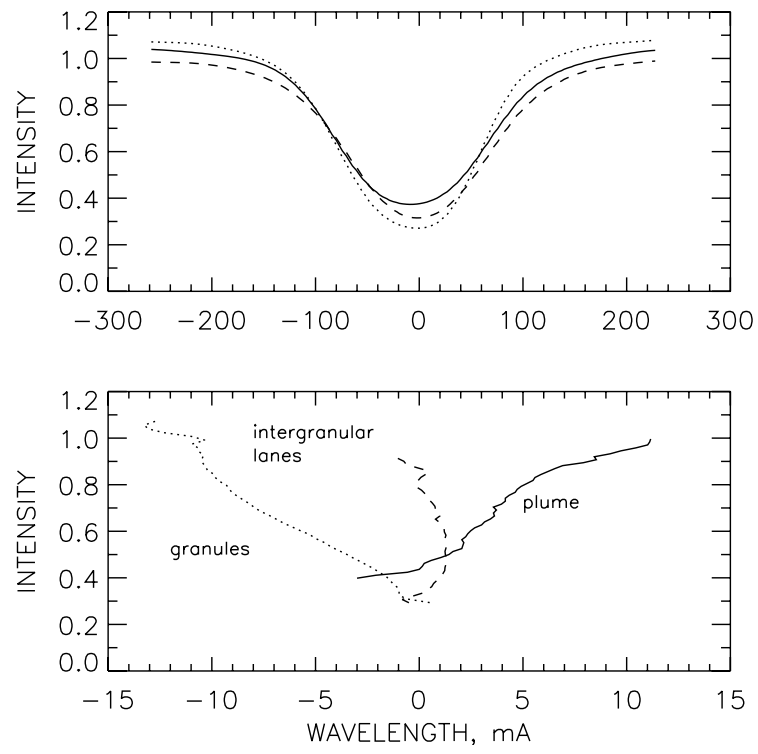

Fig. 2. Top: the average Fe I $6393 \AA$ line profiles in granules (dotted line), intergranular lanes (dashed line), and plume (solid line). Bottom: corresponding bisectors.

variations in the quiet region in the vicinity of the plume are not different from the rest of the slit positions. Note that the bright feature is absent in the bottom photosphere $(20 \mathrm{~km})$, and the granulation seems unaffected by the plume in the upper layers.

Figure 2 gives an example of the Fe I $6393 \AA$ line profiles averaged separately over granular and intergranular regions and the plume. The granular and intergranular zones were separated according to their continuum intensity in the same way as in Khomenko et al. (2001) and Kostik \& Shchukina (1999). Spectra with a continuum intensity higher (lower) than the mean continuum intensity were considered as granular (intergranular) ones. Note that, in the continuum, the intensity of the plume lies exactly in between the granular and intergranular intensity, thus making this feature indistinguishable. At the line core, the plume is significantly brighter than the average granule. The bisectors of all three features are clearly different (bottom panel of Fig. 2). The bisector of the average plume profile is shifted to the red, thus indicating the presence of a downflow.

The upper panel of Fig. 3 shows the height dependence of the intensity contrast in a quiet region and in the plume. The contrast is defined as the standard deviation of $\delta I_{l}(i, j)$ at a given level $l$, separately for the pixels containing the plume and the outside region. The plume has higher contrast above a height of about $100 \mathrm{~km}$. Its relative contrast is two times higher that that of the granulation at $H=150 \mathrm{~km}$ and is 4 times higher at $H=400 \mathrm{~km}$. The horizontal size of the feature shows a slight height dependence (bottom panel of Fig. 3). It increases from $2000 \mathrm{~km}$ at $H=100 \mathrm{~km}$ to about $2500 \mathrm{~km}$ at $H=500 \mathrm{~km}$.

\section{3. $k-\omega$ diagram}

To separate the granular and oscillatory motions, we constructed a diagnostic $k-\omega$ diagram where the power of the velocity variations appears as a function of temporal frequency $\omega$ and spatial wavenumber $k$. A detailed description of the procedure is given in Khomenko et al. (2001) and Kostik \& Shchukina (1999). The $k-\omega$ diagram shows that variations in intensity and velocity with characteristic time scales of 5 and 8-10 min are present in the plume atmosphere, similar to the quiet Sun. In the quiet Sun,
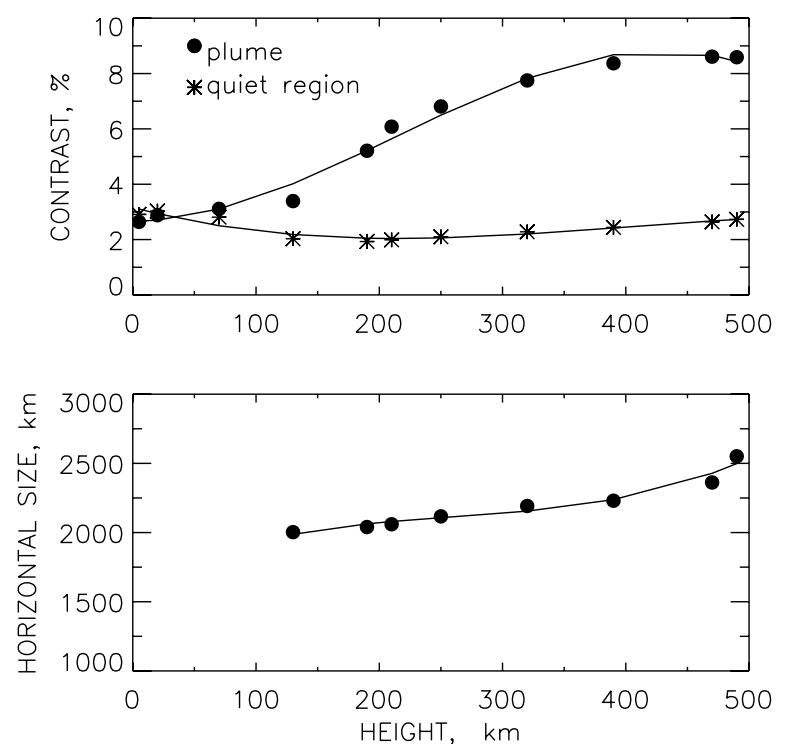

Fig. 3. Top: height dependence of the intensity contrast in a quiet region and the plume region from Fe I $6393 \AA$ line profiles. Bottom: height dependence of the horizontal size of the plume. Below $100 \mathrm{~km}$ the plume was not distinguishable from the granulation.

variations with the 5-min period are due to wave motions and variations with $8-10 \mathrm{~min}$ periods are due to convective motions. For convenience, we keep these nomenclature as referring to the plume atmosphere as well.

Based on the $k-\omega$ diagram, we restricted wave motions to the temporal frequency range $\omega=1.8-5.7 \mathrm{mHz}(T=170-560 \mathrm{~s})$ and to spatial frequency lower than $0.8 \mathrm{Mm}^{-1}$. The latter means that all the structures with sizes below 1.6 arcsec were excluded. Convective motions were restricted to temporal frequencies $\omega<$ $2.2 \mathrm{mHz}(T>450 \mathrm{~s})$. To this end we applied the corresponding low- and high-pass filters. The wave and convective motions in the plume atmosphere are studied separately in the subsequent paragraphs. For comparison, the results of a similar analysis of the quiet regions will also be shown.

\section{Dynamic conditions in the plume}

The top panel of Fig. 4 gives the mean values of the convective component of the velocity, averaged over space and time separately for granules, intergranular lanes, and the plume. Motions directed toward the observer are assumed to have a negative sign for the velocity. As expected, on average, the flow above granules is directed upwards whereas downward motions are observed above intergranular lanes. The long-period motions in the plume are more complex. At heights $H=5-200 \mathrm{~km}$, downward motions are observed, while the velocity changes its sign in the upper layers. The upward velocity above $200 \mathrm{~km}$ increases its amplitude with height, unlike the behavior of the convective velocity field in granules and intergranular lanes. No significant changes in the character of the convective motions with time inside the plume were noticed.

The middle panel of Fig. 4 shows the amplitudes of the convective component of the intensity variations (contrast) as a function of height. As expected, the average contrast of granules and intergranular lanes decreases gradually from the continuum until the temperature minimum level. Note that above $H=280 \mathrm{~km}$ granules become darker than intergranular lanes. The details of the intensity contrast inversion are discussed, for example in Kostyk \& Shchukina (2004). On contrast, the 

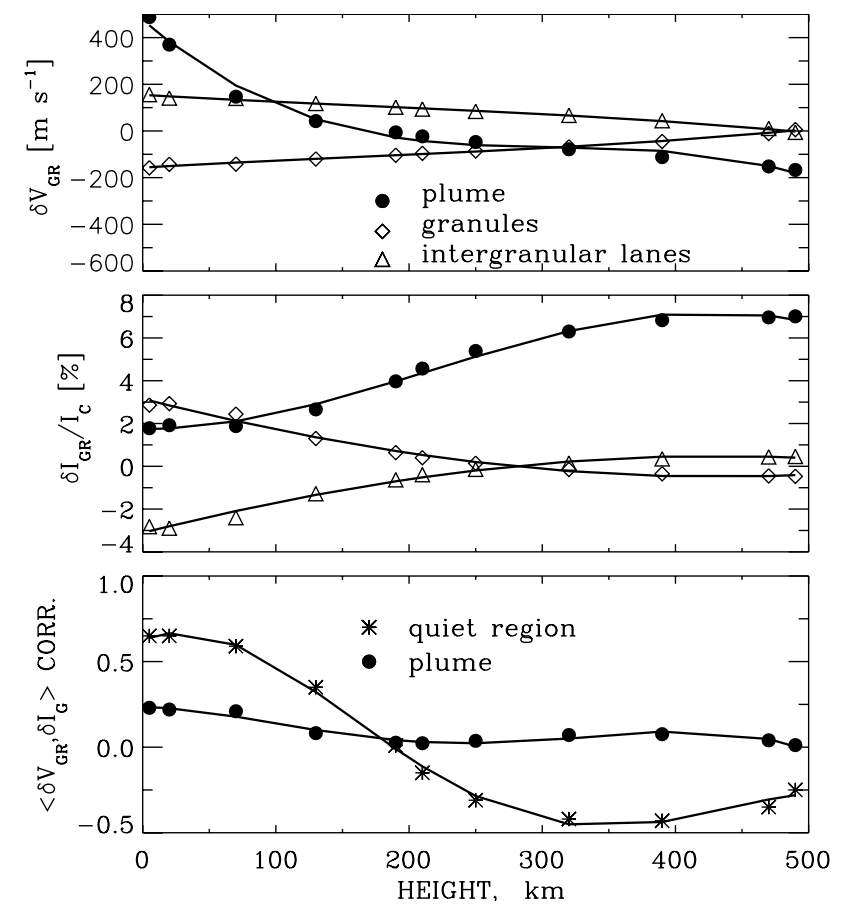

Fig. 4. Top: average values of the convective velocity as a function of height in granules (squares), intergranular lanes (triangles), and the plume (dots) from the Fe I $6393 \AA$ line profiles. The standard deviation is $25 \mathrm{~m} \mathrm{~s}^{-1}$ for the quiet region and $49 \mathrm{~m} \mathrm{~s}^{-1}$ for the plume region. Middle: amplitudes of the convective intensity variations. The standard deviation is $0.47 \%$ for the quiet region and $1.2 \%$ for the plume region. Bottom: correlation between the convective velocity and intensity variations in the quiet region (stars) and the plume (dots).

relative contrast of the plume increases with height. Such behaviour of the velocity and intensity field in the plume suggests its non-convective origin. Another confirmation of this conclusion can be found in the bottom panel of Fig. 4. It shows that the correlation between the intensity and velocity variations in the plume does not exceed 0.25 , being almost zero at heights $H=100-500 \mathrm{~km}$. This behavior should be contrasted to the typical behavior of the velocity-intensity correlation characterizing solar granulation in the quiet regions (star symbols in the bottom panel of Fig. 4).

Figure 5 compares of the properties of oscillatory motions in the plume and quiet regions averaged over space. The amplitudes of oscillations above granules and intergranular lanes were measured following the method described in Khomenko et al. (2001). Given the time variations in the oscillatory components of $\delta I_{l}(i, j)$ and $\delta V_{l}(i, j)$, the amplitudes of their extrema (maxima or minima) were identified. At the time moments where the oscillatory components have their extrema, the continuum intensity was measured. Then, the amplitudes were classified as belonging to granular or intergranular regions, depending on the value of the continuum intensity.

As follows from the top two panels of Fig. 5, the mean amplitudes of the velocity and intensity oscillations in the plume almost do not depend on height. At the same time, in the quiet area, the oscillatory amplitudes grow by a factor of 1.5-2 above granules, as well as above intergranular lanes in the given range of heights. The sign of the phase shift between the velocity oscillations at the bottom the photosphere and the upper levels (panel c of Fig. 5) corresponds to an upward wave propagation. The phase shift is greater in the plume than in the quiet area. The values of the phase shift in the quiet area are rather small; thus
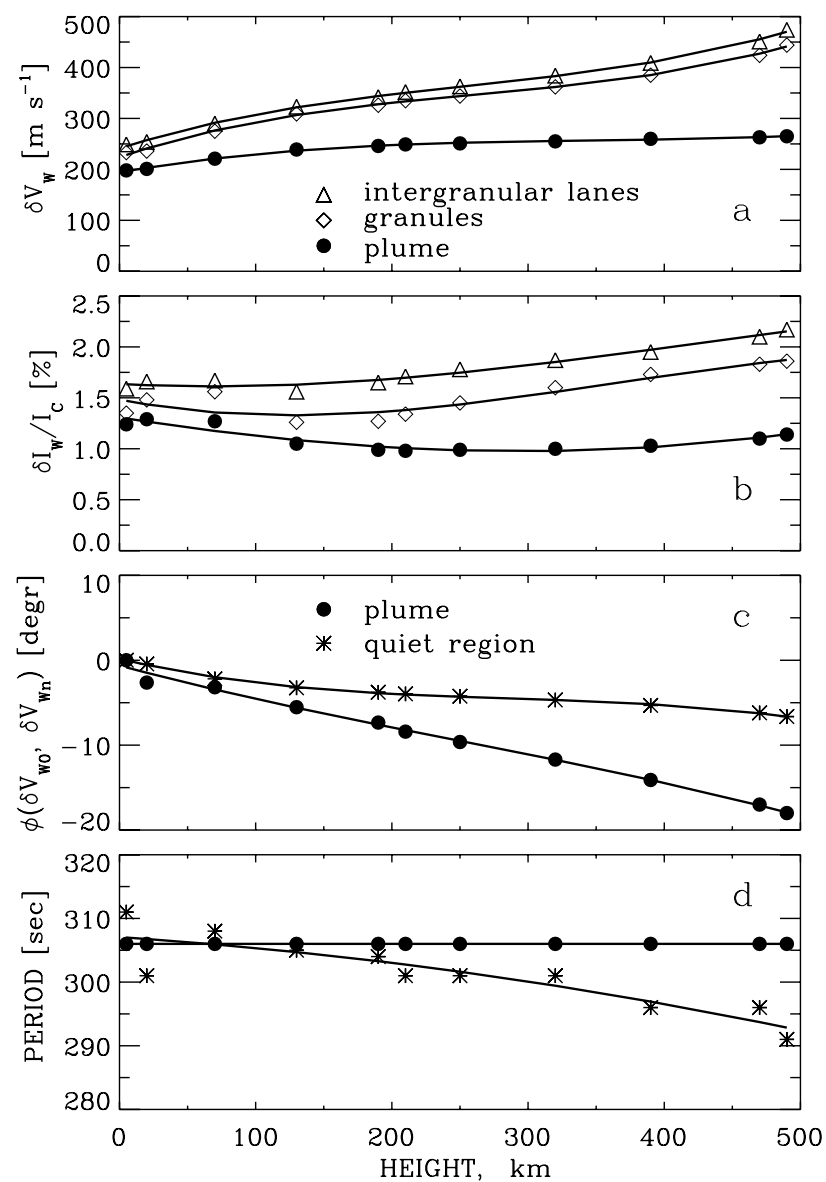

Fig. 5. a) Amplitudes of the oscillatory component of the velocity variations as a function of height in granules (squares), intergranular lanes (triangles) and the plume (dots) from the $\mathrm{Fe}$ I $6393 \AA$ line profiles. The standard deviation of the data is $47 \mathrm{~m} \mathrm{~s}^{-1}$ for the quiet region and $46 \mathrm{~m} \mathrm{~s}^{-1}$ for the plume region. b) Same for intensity variations. The standard deviation of the data is $0.20 \%$ for the quiet region and $0.15 \%$ for the plume region. c) Phase shift between velocity oscillations at $H=0 \mathrm{~km} \delta V_{W 0}$ and at higher levels $\delta V_{W n}$, in the quiet region (stars) and the plume (dots). d) Period with maximum power in the spectra in the quiet region (stars) and the plume (dots).

the waves are close to evanescent, while in the plume the waves can be considered propagating. The period of oscillations with maximum power in the spectra decreases slightly with height in the quiet area but is almost constant in the plume. The characteristic periods lie in the 5-min regime, being on average slightly larger in the plume in the upper layers. However, the difference in periods does not exceed $20 \mathrm{~s}$, and the duration of the time series does not allow conclusion as to whether this difference is statistically significant. The fact that the waves with larger periods are propagating inside the plume and waves with smaller periods are evanescent in the quiet area suggests that the thermal properties of the plume are rather different from the quiet Sun. In particular, the temperature in the plume should be higher in order to decrease the acoustic cut-off frequency and make oscillations with 5-min periods propagate.

\section{Thermal conditions in the plume}

To recover the thermal conditions in the plume, we performed an inversion of averaged Fe I 6393 and Fe II $5234 \AA$ line profiles related to granules, intergranular lanes, and the plume with 


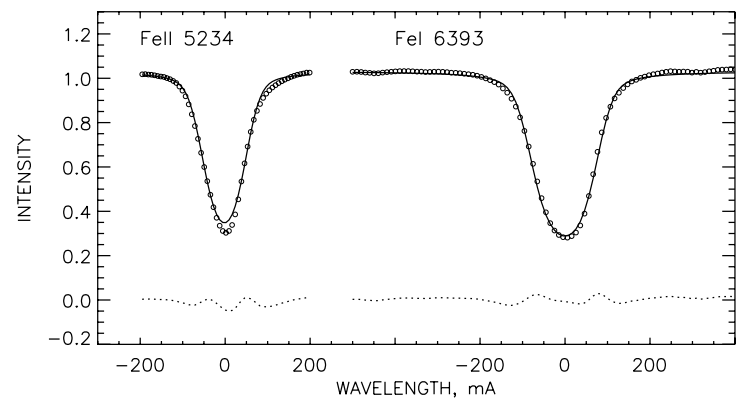

Fig. 6. An example of the fit to the average granular profile. Both Fe I 6393 and Fe II 5234 A lines are fitted simultaneously. The observed line profiles are represented by circles. The dotted line at the bottom shows the difference between observed and synthetic profiles.

the help of the SIR inversion code (Ruiz Cobo \& del Toro Iniesta 1992). We used the HSRA model as an initial model atmosphere. The free parameters of the inversion were the temperature, LOS velocity, and micro- and macroturbulent velocities. We assumed $V_{\text {mic }}$ to be constant with height and $V_{\text {LOS }}$ to have a linear dependence on height. Increasing the number of nodes for $V_{\mathrm{LOS}}$ does not qualitatively change the results, but introduces larger uncertainty since the average intensity profiles alone may not have sufficient information. The spectral lines used are sensitive to the magnetic field via the Zeeman effect since the Landé factor of the transitions is non-zero. The lines are not Zeeman triplets. The effective Landé factor $g_{\text {eff }}$ is equal to 1.64 for the Fe I $6393 \AA$ transition, and is equal to 0.8 for the Fe II $5234 \AA$ transition. However, the magnetic field strength cannot be recovered reliably just from the intensity profile if the Zeeman splitting is not complete. In the case of the Zeeman splitting being lower than the Doppler width of the profile, the broadening produced by the magnetic field cannot be distinguished from the effects of the $V_{\text {mic }}$ and $V_{\text {mac }}$, unless the later are fixed from other considerations.

We performed several tests. If $V_{\mathrm{mic}}, V_{\mathrm{mac}}$, and the magnetic field $B$ are the free parameters of the inversion, the code always converges to the same value of the $V_{\text {mic }}$, and $V_{\operatorname{mac}}$ and $B \approx 0$ independently of the choice of the initial values for these parameters. In contrast, if we fix $V_{\text {mic }}$ and $V_{\text {mac }}$ and allow the magnetic field to vary, the resulting value of $B$ depends crucially on the choice of the fixed micro- and macroturbulent velocities. The results described below are obtained by assuming $B=0$ and only velocity broadening.

The fit has the same quality for the three sets of the profiles. Figure 6 shows an example of the fit to the granular profiles. The fit in the line wings is good, being worse in the line core of the Fe II $5234 \AA$ line. The possible reason for the worse fit to the Fe II $5234 \AA$ line core is the neglect of the NLTE effects in the inversion. The strong Fe I lines, like Fe I $6393 \AA$ line, tend to be weaker if calculated under the LTE assumption (Shchukina $\&$ Trujillo Bueno 2001). If the observed profile is inverted under the assumption of LTE, this effect is compensated by a decrease of temperature. In fact, one recovers an excitation temperature that is lower than the electron temperature. The decrease in temperature leads to a decrease in the abundance of the Fe II atoms on the excited levels. The leads to shallower Fe II $5324 \AA$ line profiles than the real observed profiles.

The values of $V_{\text {mic }}$ and $V_{\text {mac }}$ obtained from the inversion are given in Table 2. $V_{\mathrm{mac}}$ is the lowest for the plume atmosphere reflecting its shallower average profile (Fig. 2). In contrast, $V_{\text {mic }}$ is highest for the plume profile, indicating its larger
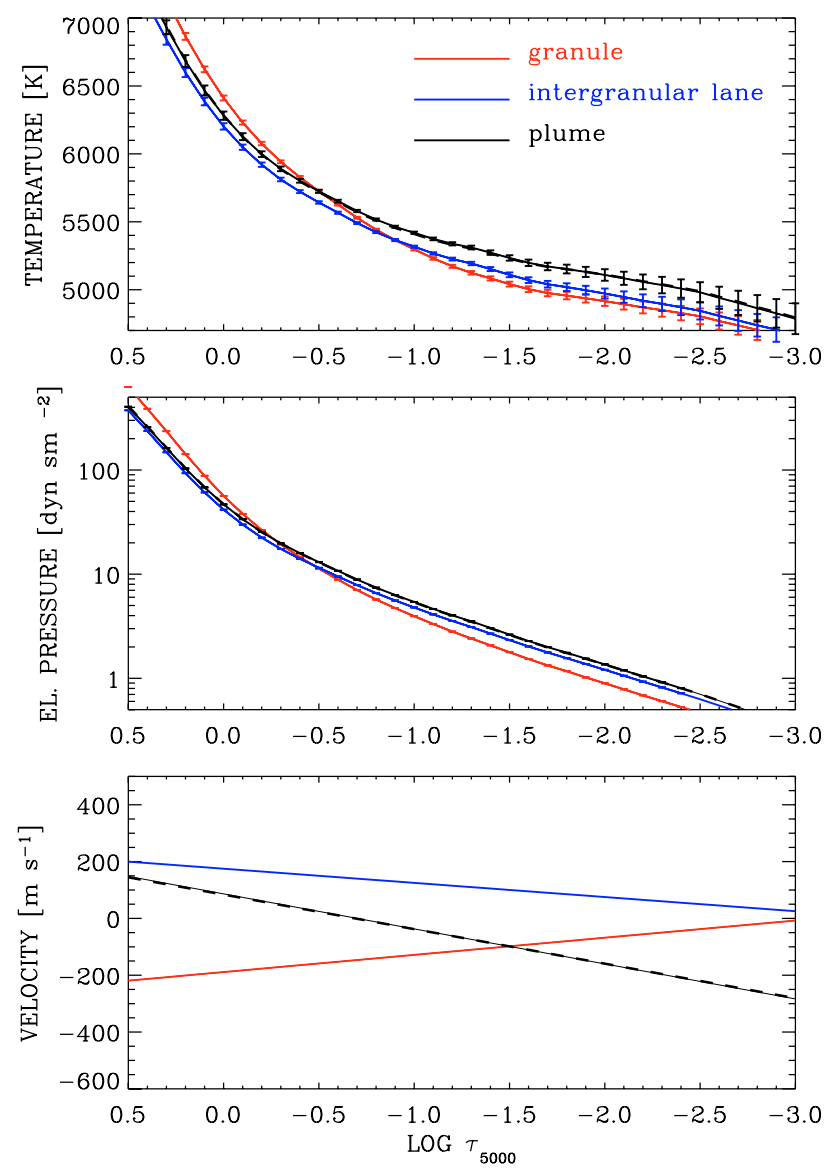

Fig. 7. Top: temperature stratifications obtained from the simultaneous inversion of the average Fe I 6393 and Fe II $5234 \AA$ line profiles in granules (red), intergranular lanes (blue), and the plume (black). The black solid line shows the results of the inversion without magnetic field, while the dashed black line shows the results of the inversion assuming a magnetic field of $400 \mathrm{G}$ filling the plume atmosphere. Middle: pressure stratification. Bottom: LOS velocity. For clarity, we do not show the error bars for the LOS velocity. The errors make $\pm 140 \mathrm{~m} \mathrm{~s}^{-1}$ for granules, $\pm 207 \mathrm{~m} \mathrm{~s}^{-1}$ for intergranular lanes, and $\pm 261 \mathrm{~m} \mathrm{~s}^{-1}$ for the plume at all heights.

Table 2. Micro- and macroturbulent velocities obtained from the inversion.

\begin{tabular}{cccc}
\hline \hline & Granules & Intergranular lanes & Plume \\
\hline$V_{\text {mic }}\left[\mathrm{km} \mathrm{s}^{-1}\right]$ & $1.3 \pm 0.1$ & $1.5 \pm 0.1$ & $1.7 \pm 0.1$ \\
$V_{\text {mac }}\left[\mathrm{km} \mathrm{s}^{-1}\right]$ & $1.1 \pm 0.1$ & $1.0 \pm 0.2$ & $0.9 \pm 0.3$ \\
\hline
\end{tabular}

width. Possibly, the broadening of the profile in the plume is produced by the presence of the magnetic field. However, the inversion procedure does not allow us to distinguish between the effects of the magnetic field and turbulent velocities.

Figure 7 gives the resulting stratifications of temperature, electron pressure, and LOS velocity obtained from the inversion as a function of the optical depth $\tau_{5000}$. In the deep layers, granules are hotter than intergranular lanes. The temperature inversion is observed about $\log \tau_{5000}=-1$. Above that depth, intergranular lanes become hotter. The temperature in the plume stays between the granular and intergranular temperatures in the deep layers, indicating that this feature cannot be distinguished from the average atmosphere. In the upper atmosphere, the plume is about $100-200 \mathrm{~K}$ hotter than the quiet atmosphere at a given $\tau_{5000}$, which explains its brightness in Fig. 1 . The 
electron pressure behaves like the temperature. Note that the velocity stratifications obtained from the inversion agree with those obtained from the $\lambda$-meter method (Fig. 4), confirming the validity of the results.

As our data include only unpolarized spectra, we cannot discard the possibility that the observed plume is a magnetic feature. In this case one may argue that the magnetic field could, in principle, affect our results for the thermal stratification and velocity distribution. To discard this possibility, we performed an additional inversion test. All the parameters of the inversion were the same as described above, except that we forced the magnetic field to $400 \mathrm{G}$, filling all the volume (filling factor 100\%). This value is reasonable in view of the small-scale magnetic elements in the chromospheric network having an intrinsic field strength of $1-2 \mathrm{kG}$ and filling $10-20 \%$ of the volume. The results of this inversion test are shown in Fig. 7. The resulting stratification of the temperature, pressure, and velocity did not change assuming the magnetic field broadening of the profile instead of the velocity broadening. This proves the validity of our results. As expected, the resulting values of $V_{\text {mic }}$ and $V_{\text {mac }}$ have changed slightly compared to the non-magnetic case, $V_{\text {mic }}=1.6 \mathrm{~km} \mathrm{~s}^{-1}$, and $V_{\mathrm{mac}}=0.8 \mathrm{~km} \mathrm{~s}^{-1}$. Note that in the case where the plume is a magnetic feature, the stratifications of the temperature, pressure, and velocity shown in Fig. 7 can be understood as the mean values between the magnetized atmosphere and its non-magnetic surroundings.

\section{Theoretical model of oscillations}

Can the difference in the thermodynamic structure explain the difference in the oscillatory amplitudes between the plume and the quiet regions (Fig. 5)? To check this, we computed the amplitudes of the velocity oscillations for the $300 \mathrm{~s}$ period wave propagating vertically in a stratified atmosphere (see Mihalas \& Toomre 1982; Mihalas \& Mihalas 1984; Marmolino et al. 1993). Following Khomenko et al. (2001), the atmosphere was divided into a series of isothermal layers in order to integrate the wave equation. In each of them the velocity has the following dependence on height:

$V_{1 Z}(z, t)=V_{0} \exp \left(-\frac{z}{2 H_{\mathrm{p}}}\right) \exp \left[\mathrm{i}\left(\omega t+k_{z} z\right)\right]$

where $H_{\mathrm{p}}=P_{0} /\left(\mathrm{d} P_{0} / \mathrm{d} z\right)$ is the pressure scale height and the vertical wave number $k_{z}$ is found from the dispersion relation:

$k_{z}^{2} C_{\mathrm{s}}^{2}-\omega^{2} \frac{\gamma}{\gamma_{*}}+\frac{C_{\mathrm{s}}^{2}}{4 H_{\mathrm{p}}^{2}}=0$.

In the latter equation, the parameter $\gamma_{*}$ is equal to

$\gamma^{*}=\frac{\mathrm{i} \gamma \omega+1 / \tau_{R}}{\mathrm{i} \omega+1 / \tau_{R}}$

where $\tau_{R}$ is the radiative relaxation time of the temperature perturbations calculated according to Spiegel (1957). The initial velocity amplitude $V_{0}$ at the first isothermal layer was taken from observations at $H=0 \mathrm{~km}$ (see Fig. 5). The temperature and pressure stratifications were taken from the inversion of the profiles in granules, intergranular lanes, and the plume.

The amplitudes of the velocity oscillations calculated according to Eq. (1) are displayed in Fig. 8. The height dependence of the oscillatory amplitudes in the quiet regions (granules and intergranular lanes) agree closely with the observed

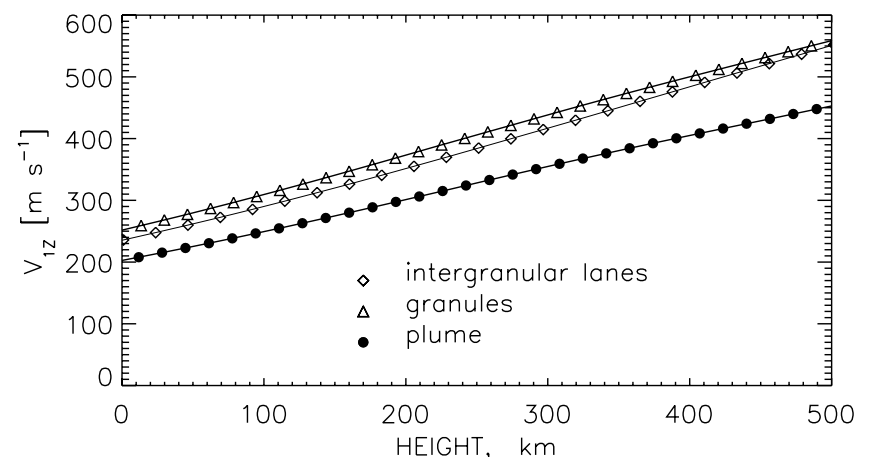

Fig. 8. Amplitudes of the vertical velocity oscillations with a $300 \mathrm{~s}$ period, as calculated from Eq. (1) using the model atmosphere obtained from the inversions in granules, intergranular lanes, and the plume.

ones (Fig. 5). The amplitudes of oscillations above intergranular lanes are larger at all heights. As for the plume, there is no agreement between the amplitudes of oscillations obtained from observations and from Eq. (1). It suggests that the theory of acoustic-gravity oscillations in a non-magnetic atmosphere is not appropriate for explaining the oscillations is this structure. Several mechanisms can be responsible for damping the wave amplitude in the plume, as we discuss below.

\section{Discussion and conclusions}

In this paper we have analyzed the thermal and dynamical properties of a bright long-lasting feature observed in solar granulation. Both convective and oscillatory motions turned out to be different inside the plume compared to the nearby quiet atmosphere. The waves change their period, propagation speed, and the amplitude dependence on height. The differences in the convective flows, intensity contrast, and the absence of the velocityintensity correlation, characteristic of the granulation, suggest a non-convective origin of the feature. But then what is the origin of the plume?

Espagnet et al. (1995) and Roudier et al. (2001) mention bright plums observed in solar granulation in the time series of MSDP observations in Na I $\mathrm{D}_{2}$ line at $5890 \AA$. The authors call such a features "bright sinking plumes" due to the apparent motion of the features from the top to the bottom of the photosphere. According to Espagnet et al. (1995), the relative contrast of the "plumes" increases with height and reaches a maximum at heights of $250-500 \mathrm{~km}$ and a large-scale flow inside these features is directed downwards. About 10 such events were detected in an area of $8^{\prime \prime} \times 172^{\prime \prime}$ with $80 \%$ of their lifetimes exceeding 16 min (the duration of the time series used by Espagnet et al. 1995) and sizes varying from 1 to 4 arcsec. The authors suggest that the plumes have neither a convective nor magnetic origin but instead originate in the chromosphere. Many of the plums are located at the edges of exploding granules (Roudier et al. 2001). Unfortunately, our observations are done with a fixed slit position and do not allow study of the morphology of the granulation in the vicinity of the plume. Espagnet et al. (1995) reported that the plume appears first in the upper photosphere and extends downwards as its contrast increases. Despite the much longer duration of our observation in comparison to that of Espagnet et al. (1995) (158 min compared to $16 \mathrm{~min}$ ), no clear changes in the character of the velocity or intensity variations in the plume were observed. The upflows in high layers observed by us also 
contrast to the results of Espagnet et al. (1995). Thus, it is not clear whether the same classes of the phenomena were detected.

The size of the plume of 3-4 arcsec makes it close to a solar pore or a magnetic knot (Spruit \& Zwaan 1981; Soltau 1997; Tritschler \& Uitenbroek 2006). However, these features appear dark at the continuum wavelength, while the plume cannot be distinguished from the nearby granulation. The brightness of the plume increases with height, making it closer to the behavior of small-scale magnetic features such as network flux tubes. However, the flux tubes have a typical size of a fraction of an arcsec. The spatial resolution of our observations of 1 arcsec does not allow us to resolve such structures. The seeing would make the bright points increase their linear size, but would not make them as large as 3-4 arcsec. These arguments do not exclude the possibility that a large conglomerate of bright flux tubes was observed. As appears in Soltau (1997), the 1 arcsec seeing is sufficient for making individual small-scale structures with similar properties located close together to be spatially unresolved so as to appear as a single structure. Unfortunately, the quality of the Ca II K slit-jaw images was insufficient for confirming or discarding this possibility. Since we deal with non-polarimetric data, the magnetic field strength in the observed feature also could not be extracted with sufficient reliability from the observed line profiles. However, there are several indirect hints of the magnetic origin of the plume. First, an increase of the linear size of the feature with height (Fig. 3, bottom panel) may be interpreted as an indication that magnetic field lines fan out with height in a magnetic flux tube. Second, an enhanced microturbulent velocity in the plume may suggest a magnetic broadening of the profiles. Finally, the behavior of oscillations in the plume is clearly different from that in the quiet atmosphere, and we have shown that the differences in the thermal stratification in the plume cannot take account of this observational fact.

The presence of the magnetic field can modify the properties of the waves in the plume. Assume the plume is a magnetic structure. According to Fig. 1, its spatial location does not change with height. It means that the assumption of a vertical field can be made. It is known that vertically propagating waves in a vertical magnetic field are not different from classical acoustic waves (Ferraro \& Plumpton 1958), so the magnetic field cannot be responsible for the reduction of the wave amplitude. However, if the direction of the propagation of a quiet Sun acoustic wave is different from the vertical, the mode transformation could occur in the case of the presence of a magnetic field in the region where the acoustic speed is equal to the Alfvén speed. Due to mode transformation, a part of the energy of an acoustic wave can go into other wave types, such as Alfvén waves or other types of flux tube modes. These modes are transversal and produce variations in the horizontal velocity component. Variations in the horizontal velocity will remain undetected in observations of the solar disc center. Since the mode transformation extracts some part of the energy of the vertical velocity variations, this would produce an apparent decrease in the wave amplitude detected in observations.

Independent of the nature of the plume, an important finding is the presence of propagating waves in this feature in contrast to the evanescent waves in the quiet Sun. It is important to know that five-minute oscillations can be channeled from the photosphere to the upper layers by vertical small-scale structures, no matter what their nature. The wave channeling by vertical network elements has been reported recently by Vecchio et al. (2007) from observations obtained with the IBIS instrument. The observational confirmation of five-minute wave propagation from the bottom to the top photosphere in small-scale vertical structures is important for understanding the mechanisms of wave energy transport to the upper atmosphere.

Acknowledgements. This work was partially supported by the Spanish Ministerio de Educación y Ciencia through project AYA2004-05792.

\section{References}

Beckers, J. M., \& Schröter, E. H. 1968, Sol. Phys., 4, 142

Berger, T. E., \& Title, A. M. 2001, ApJ, 553, 449

Berger, T. E., Rouppe van der Voort, L. H. M., Löfdahl, M. G., et al. 2004, A\&A, 428,613

Deubner, F. L. 1989, A\&A, 216, 259

Espagnet, O., Muller, R., Roudier, T., et al. 1995, A\&AS, 109, 79

Ferraro, V. C. A., \& Plumpton, C. 1958, ApJ, 127, 459

Gurtovenko, E. A., \& Kostik, R. I. 1989, Fraunhofer Spectrum and the System of Solar oscillator Strengths, Russian Language Edition, Naukova Dumka, Kiev

Khomenko, E. V., Kostik, R. I., \& Shchukina, N. G. 2001, A\&A, 369, 660

Kostik, R., \& Shchukina, N. 1999, Astron. Lett., 25(10), 678

Kostyk, R., \& Shchukina, N. 2004, Astron. Rep., 48, 769

Leighton, R. W., Noyes, R. W., \& Simon, G. W. 1962, ApJ, 135, 474

Maltby, P., Avrett, E. H., Carlsson, M., et al. 1986, ApJ, 306, 284

Marmolino, C., Severino, G., Deubner, F.-L., \& Fleck, B. 1993, A\&A, 278, 617

Mihalas, B. W., \& Toomre, J. 1982, ApJ, 263, 386

Mihalas, D., \& Mihalas, B. W. 1984, Foundations of Radiation Hydrodynamics (Oxford: Oxford University Press)

November, L. J., Toomre, J., Gebbie, K. B., \& Simon, G. W. 1981, ApJ, 245, L123

Roudier, T., Eibe, M. T., Malherbe, J. M., et al. 2001, A\&A, 368, 652

Ruiz Cobo, B., \& del Toro Iniesta, J. C. 1992, ApJ, 398, 375

Shchukina, N. G., \& Trujillo Bueno, J. 2001, ApJ, 550, 970

Shelyag, S., Schüssler, M., Solanki, S. K., Berdyugina, S. V., \& Vögler, A. 2004, A\&A, 427, 335

Sobotka, M., Bonet, J. A., \& Vázquez, M. 1994, ApJ, 426, 404

Soltau, D. 1997, A\&A, 317, 586

Spiegel, E. A. 1957, ApJ, 126, 202

Spruit, H. C., \& Zwaan, C. 1981, Sol. Phys., 70, 207

Stangl, S., \& Hirzberger, J. 2005, A\&A, 432, 319

Stebbins, R. T., \& Goode, P. R. 1987, Sol. Phys., 110, 237

Stein, R. F., Carlsson, M., Nordlund, A., \& Sharmer, G. 2004, in Am. Astron. Soc. Meet., 204, 88.04

Title, A. M., Tarbell, T. D., Topka, K. P., et al. 1989, ApJ, 336, 475

Tritschler, A., \& Uitenbroek, H. 2006, ApJ, 648, 741

Vecchio, A., Gauzzi, G., Reardon, K. P., Janssen, K., \& Rimmele, T. 2007, A\&A, 661, L1 